Ján DEMČIŠÁK

https://orcid.org/0000-0003-0227-6716

Simona FRAŠTÍKOVÁ

https://orcid.org/0000-0001-7699-3756

Univerzita sv. Cyrila a Metoda (Trnava)

\title{
Effekte der Affekte. Das politische Spiel mit dem Gemüt des Rezipienten
}

\section{Effects of affects. The political game with the feelings of the recipient}

\begin{abstract}
The increasingly louder voice of right-wing populist discourse undoubtedly deserves our attention. In this context, the question arises: what helps the different right-wing populist subjects to achieve their success? How and how do they address the voters to win their vote? The paper deals with the techniques and strategies of political manipulation, especially with the technique of emotionalization. The focus is on two opposite poles on the emotional or affective scale, which can be associated with grief and crying or laughing and pleasure. The research deals with the relevance that the visual material (posters, online presence, etc.) used by the political parties has on the voter recipients. The study analyzes which effects result from the representation and instrumentalization of affects and emotions and how they can influence the recipient.

Keywords: right-wing populism, political poster, pity, grief, crying, laughing, political joke, caricature, political manipulation.
\end{abstract}




\section{Vorspiel}

Die Macht der visuellen Mittel liegt in ihrer Kraft, Aufmerksamkeit zu erregen, Neugier zu wecken, zu verführen, zu provozieren, aber auch beruhigen zu können und nicht selten sogar den Betrachter zu einer gewissen Gegenreaktion zu bringen. Sie gehören zu den wirkungsvollsten Formen der Kommunikation, indem sie es vermögen, direkt im Moment ihrer Betrachtung die unterschiedlichsten Emotionen und Affekte zu entfachen, wodurch sich die vermittelten inhaltlichen Botschaften besser merken bzw. mit den jeweiligen Emotionen verbinden und aufgrund dessen auch beurteilen lassen.

Das große Potenzial der visuellen Mittel im Rahmen der Kommunikation demonstrieren vielfältige Einsatzmöglichkeiten wie auch unterschiedliche Bereiche sowohl des privaten als auch des öffentlichen Lebens, in denen sie regelmäßig und bewusst aufgegriffen werden. Zweifelsohne gehört dazu ihr Gebrauch in der Sphäre des politischen Marketings. Gezielte Kommunikation mit dem potenziellen Wähler und eine raffiniert gewählte Ansprache des unzufriedenen Bürgers haben dem Populismus und mithin den rechtspopulistischen Parteien sicherlich zu wachsender Popularität und Wahlerfolgen verholfen.

Dass sich die Rechtspopulisten einer besonderen Aufmerksamkeit erfreuen, konnte dem Blick der gegenwärtigen Forschung sicherlich nicht entgehen. Bisweilen wurde eine ganze Reihe an typischen Merkmalen beschrieben und charakterisiert, die das Wesen des Populismus ausmachen, von denen hier nur einige stichwortartig genannt werden sollen: ${ }^{1}$

- Berufung auf (einfachen) gesunden Menschenverstand und common sense,

- Anti-Establishment (Anti-Elitarismus, Anti-Intellektualismus),

- „Wir“-Perspektive statt „Ich“-Perspektive,

- religiöser, ethnischer, kultureller Patriotismus: „wir, unser Land“ vs. „andere, Fremde",

- starke Thematisierung der Europäischen Flüchtlingskrise (gruppenbezogene Menschenfeindlichkeit, Kritik an der EU),

1 Vgl. u. a. Frank Decker, Marcel Lewandowsky, Rechtspopulismus: Erscheinungsformen, Ursachen und Gegenstrategien (Bonn: BPB, 2017), https://www.bpb.de/politik/extremismus/rechtspopulismus/240089/rechts-populismuserscheinungsformen-ursachen-undgegenstrategien; Karin Priester, „Wesensmerkmale des Populismus“, Aus Politik und Zeitgeschichte 62 (Bonn: BPB, 2012): 3-9; Karin Priester, „Das Syndrom des Populismus“, Dossier: Rechtspopulismus (Bonn: BPB, 2017): 7-12, www.bpb.de/system/files/ pdf_pdflib/pdflib-241384.pdf; Tim Spier, „Populismus und Modernisierung,“ in Populismus, hrsg. v. Frank Decker (Wiesbaden: VS Verlag für Sozialwissenschaften, 2006), 33-58; Paul Taggart, Populism. Buckingham: Open University Press, PA, 2000. 
- charismatische Führungspersönlichkeit,

- Thematisierung der Gerechtigkeit,

- besonderes Engagement für Familien- und Sozialpolitik,

- Polarisierung als eine der dominanten Thematisierungsstrategien.

Hinsichtlich der aufgezählten Merkmale ist festzustellen, dass sie ein relativ breites Spektrum an verschiedenen (Teil)Aspekten zur Thematisierung auch im Zusammenhang mit dem analysierten Bildmaterial bieten. Den untersuchten Bildkorpus stellen insbesondere Billboards, Flyer, Grafiken, Karikaturen und die auf Online-Plattformen und in sozialen Medien veröffentlichten Bild- oder Bild-Text-Beiträge. Die gesamte Materialbasis fällt in den Zeitraum von 2013 bis $2018 .^{2}$

In Zusammenhang mit der Analyse des visuellen Materials und unter der Berücksichtigung des Erfolgs der rechtspopulistischen Kommunikationsstrategien können zwei Fragen aufgeworfen werden:

- Was und wie wird anhand von visuellen Mitteln dargestellt? Warum und mit welcher Intention wurde diese und jene Darstellungsform verwendet?

- Wie und mit welcher Wirkung wird das Dargestellte wahrgenommen?

Es ist offensichtlich, dass es hier um zwei unterschiedliche Perspektiven geht, die des Produzenten und die des Rezipienten, die jedoch bei der komplexen Analyse nicht zu trennen sind. Im vorliegenden Beitrag liegt die Konzentration primär auf der durch die Wähler vertretene Rezipientenperspektive, nichtsdestotrotz lässt sich die des Produzenten nicht ganz außer Acht lassen, denn die Motivation für die mit visuellen Mitteln vermittelten Inhalte kann erst vor dem Hintergrund inhaltlicher Schwerpunkte und typischer Betätigungsfelder des Produzenten, hier der rechtspopulistischen Parteien in Deutschland, Österreich, Tschechien und der Slowakei, erörtert werden.

Bei der Beachtung der Rezipientenperspektive spielt das Emotionale eine wichtige Rolle. Bei der unmittelbaren Betrachtung einer bildlichen Darstellung, etwa eines Fotos oder einer Zeichnung, werden zuerst bestimmte Gefühle und Assoziationen ausgelöst, welche die anschließende Beurteilung und das Handeln des Rezipienten beeinflussen können. Die Frage, ob eine durch visuelle Mittel oder durch eine Bild-Text-Kombination vermittelte Botschaft den Rezipienten zum „Lachen“ oder zum „Weinen“ gebracht hat oder bringt, lässt sich kaum beantworten. Ausgehend von Assoziationen, die mit diesen Begriffen verbunden sind (etwas Positives, Lustiges vs. etwas Negatives, Trauriges), können wir sehr gut das durch visuelle Mittel oder eine

2 Die Bild- und Text-Datenbank als auch diese Analyse entstehen im Rahmen des Projekts APVV-17-0128 Language strategies of right-wing populists in comparation (Germany - Austria - Czech Republik - Slovakia): lexis - texts - discourses. 
Bild-Text-Kombination Mitgeteilte auf einer Skala der potenziell zu den Effekten „Lachen" und „Weinen“ führenden Emotionen markieren. Vereinfacht gesagt konzentrieren wir uns auf das emotionale Spiel mit der Trauer und der Lust und beschäftigen uns zugleich mit dessen Folgen, wobei die Anspielungen auf das klassische Vokabular der Dramentheorie nicht zufällig gewählt sind und unsere spezifische Rezeptionssicht verdeutlichen sollen.

Infolgedessen, dass bei der allgemeinen Charakteristik der Wesenszüge des Rechtspopulismus eher negative Aspekte genannt werden, wird an dieser Stelle mit dem Auslösen von überwiegend negativen und schaurigen Emotionen gerechnet. Diese sind auch als die ersten unter die Lupe zu nehmen.

\section{Effekte des Spiels mit Rührung und Trauer}

„Weinen ist [...] ein Mehrzweckverhalten.“" Wer weint, kann traurig, verzweifelt, wütend, oder auch gerührt oder glücklich sein. Weinen lässt somit sowohl eine positive als auch eine negative Deutung zu, je nach seinem Anlass und seiner anschließenden Betrachtung durch den Empfänger. Da die reizauslösenden Motive subjektiv bewertet werden, können sie Auslöser sowohl positiver als auch negativer Emotionen sein. Im Anschluss daran wird auf die Analyse der empirischen Materialbasis eingegangen, indem wir uns, wie oben angedeutet, in die Rolle des Rezipienten versetzen und versuchen, den exemplifizierenden Anlässen die durch sie auszulösenden Emotionen zuzuordnen. Inwieweit die eventuell ausgelösten Emotionen Weinen zur Folge haben, lässt sich nur schwer beantworten. Statt zu generalisieren, lassen wir diese Frage lieber offen.

Die Thematisierung von Sachverhalten im politischen Diskurs erfolgt anhand von unterschiedlichen Darstellungsstrategien. Auch identische inhaltliche Schwerpunkte können vor dem Hintergrund der verfolgten Absicht auf zweierlei Art und Weise abgebildet werden. Mit Rücksicht auf Weinen als einen denkbaren Effekt interessieren uns Bilder mit dem Potenzial, in uns Rührung, Trauer oder Schauer zu erwecken. Ein typisches Merkmal dieser Bilder ist in der Regel ein realer Akteur, darunter je nach dem thematisierten Sachverhalt entweder jemand, mit dem sich der Rezipient identifizieren kann oder eine inkriminierte Person bzw. Personengruppe (nicht akzeptierte, nicht anpassungsfähige Personen oder Kriminelle), von denen er Abstand hält.

Durch die absichtliche Wahl eines zur Identifikation einladenden Akteurs werden zwei Auffassungspositionen des (Wahl)Mittels geschaffen; die

3 Vgl. Lexikon der Biologie [Stichwort:] „Weinen“, https://www.spektrum.de/lexikon/ biologie/weinen/70503. 
eine tangiert den Blick von außen, das heißt wie wirkt das Abb. direkt im Moment der Betrachtung, und die andere zieht den Betrachter ins Bild hinein, sodass er sich betroffen fühlt. Paradebeispiele dafür sind die Abbildungen 1 und 2, die ein wichtiges politisches und soziales Thema behandeln, nämlich Rentner und ihre Position im Sozialsystem.

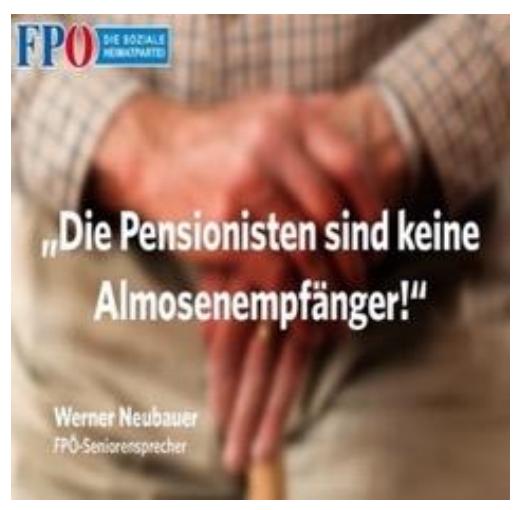

Abb. 1

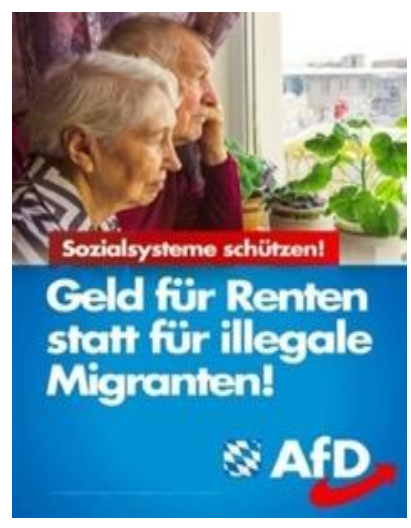

Abb. 2

Das dominante visuelle Mittel beider Bilder sind Rentner. Bei der ersten Abbildung geht es nur um einen Ausschnitt aus der Aufnahme eines sich auf einen Stock stützenden Rentners, wobei die den Stock haltenden Hände im Vordergrundstehen. Der Stock kann hier als Symbol der Unterstützung auch im übertragenen Sinne des Wortes verstanden werden. In der zweiten Abbildung sitzt ein Rentnerpaar am Fenster, schaut ziellos nach draußen, freudlos und eher sorgenvoll. Von außen, aus einer gewissen Distanz, erwecken beide Bilder das positiv konnotierte Gefühl des Mitleids mit „schwächeren" sozialen Gruppen (kraftlose Rentner, sozial benachteiligte Familien u. ä.), die auf jemandes Hilfe angewiesen sind. Infolge der Einbeziehung des Betrachters ins thematisierte Geschehen entstehen weitere, jedoch negative Konnotation aufweisende Emotionen: Angst vor der eigenen Situation in der entsprechenden Lebensetappe, Ärger über die Tatenlosigkeit der herrschenden politischen Parteien für diese Zielgruppe (mithin potenziell auch für den Betrachter und seine Nächsten) und schließlich das daraus resultierende Misstrauen gegen das politische System. Diese Emotionen weisen darauf hin, dass der Betrachter sich selbst in die Situation der abgebildeten Rentner hineinprojiziert.

Auf ähnliche Weise demonstriert auch die folgende Bilderreihe das Gefühl des Mitleids als unmittelbare Reaktion auf einen visuell dargebotenen Inhalt. 


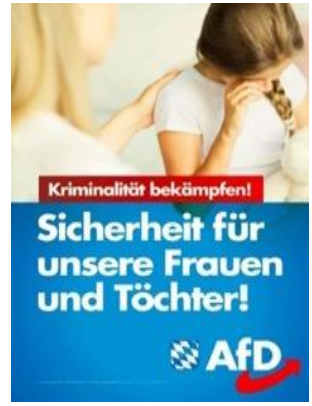

Abb. 3

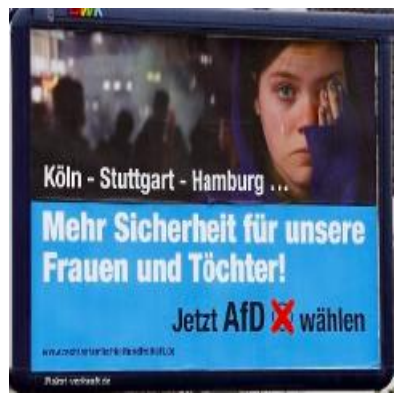

Abb. 5

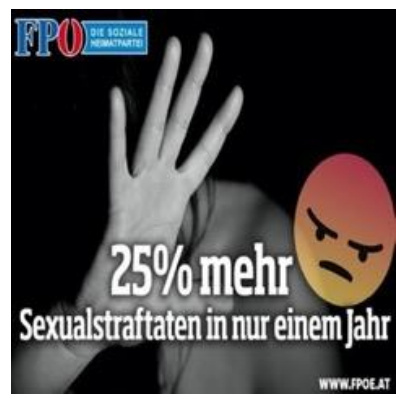

Abb. 7

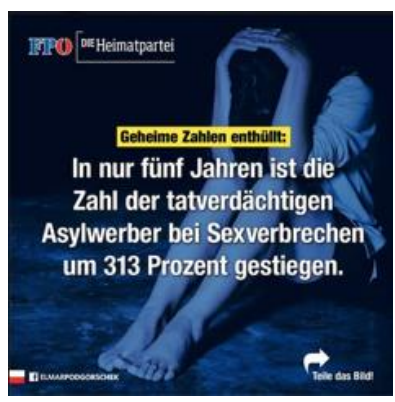

Abb. 9

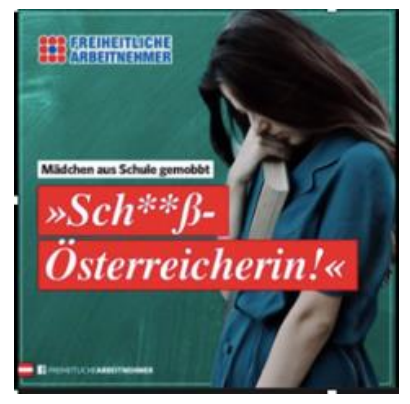

Abb. 4

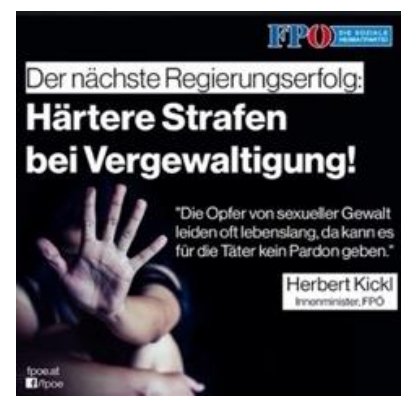

Abb. 6

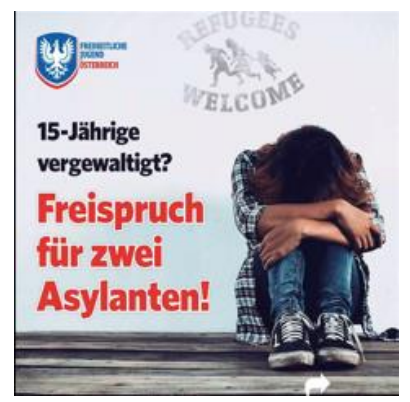

Abb. 8

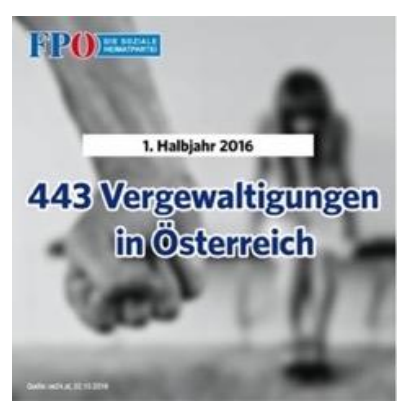

Abb. 10 
Die Kraft der angeführten Bilder liegt bereits in der Wahl eines heiklen Themas, das angesprochen werden soll. Das Gefühl des Mitleids entsteht hier im Bezug auf Opfer von Aggression und Vergewaltigung, vertreten durch Akteurinnen überwiegend jüngeren Alters. In der gesamten Bilderreihe ist die Beklommenheit dieser Akteure zu spüren, die sich anhand ihrer Körpersprache, besonders der Haltung der Hände und der Blickrichtung erschließt. Auf den einzelnen Bildern wird sie von weiteren Gefühlen begleitet, dabei von Angst vor Wiederholung solcher Erfahrungen (Abb. 1: Das Mädchen schaut zu Boden und wischt sich Tränen aus den Augen; es hält ein Kuscheltier als eine Art gewissen emotionalen Halts oder Hilfe; Abb. 5: Tränen, Blick voller Angst verbunden mit dem Blick auf eine dunkle nächtliche Straße im Hintergrund, angespannter Mund des Mädchens; Abb. 6-7: abwehrende Stopp-Handhaltung der Opfer; Abb. 10: geballte Faust als Symbol der Gewalt, das auf das Schlimmste wartende, zu Boden schauende Mädchen), Unsicherheit über die Weiterentwicklung dieser oder ähnlicher Geschehnisse (Abb. 3-10), Schande und Demütigung im Sinne von „Jetzt zeigen andere/alle auf mich!" (Abb. 3: Das Mädchen schaut zu Boden und wischt sich Tränen aus den Augen; Abb. 4: Das an der Tafel stehende Mädchen als Objekt der Verspottung und Verachtung, Blick zu Boden, angespannter Mund; Abb. 89: Die Opfer sitzen gebeugt und den Kopf trübselig in die Hände gelegt auf dem Boden), Schuld auf der Mutter- (Abb. 3: „Hätte ich sie nicht mehr schützen können?") oder Opfer-Seite (Abb. 4: „Was habe ich falsch gemacht?“/„Warum gerade ich?"). Bei der Einschätzung dieser Plakate kann das Geschlecht des Betrachters eine wichtige Rolle spielen. Frauen könnten auf den dargestellten Inhalt sensibler reagieren, indem sie sich selbst oder eventuell ihre eigenen Töchter als potenzielle Opfer sehen. Bei Männern hingegen könnte ihre Rolle als Helfer oder Beschützer in den Vordergrund treten.

Mit dem Gefühl des Mitleids sind nicht zuletzt auch die Abbildungen 11 und 12 verbunden.

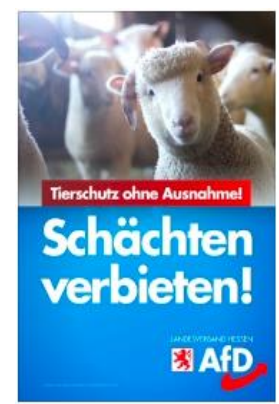

Abb. 11

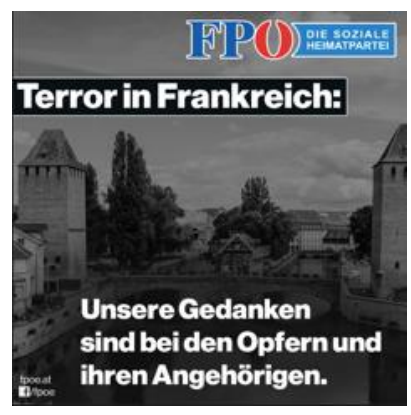

Abb. 12 
Sie bilden völlig unterschiedliche Inhalte ab, nämlich Tierschutz und Terrorismus, die sich erst vor dem Hintergrund der begleitenden Texte richtig verstehen lassen. Beim Thema Tierschutz steht im Mittelpunkt das neugierig und fast naiv schauende Schaf, das vom Wesen her ein ängstliches Tier ist. Im anderen Fall kann als eine Art Impuls für die Auslösung des Mitgefühls die Farbgestaltung des Fotos erkannt werden. Das graue Foto und die düstere Atmosphäre evozieren Melancholie und Trauer. Beide Bilder nehmen zugleich direkt oder indirekt Bezug auf Religiosität. Sowohl die unakzeptable Vorstellung des rituellen Schlachtens von Tieren als auch die Assoziation des Terrorismus mit dem Islam evozieren Unbehagen und Mitleid bzw. Trauer und schlagen eventuell in Angst vor anderen Religionen um.

Die Interpretation des Mitleids in Richtung seiner positiven Bedeutung wäre normalerweise auch im Anschluss auf die Wahrnehmung von Bildern wie die Abbildungen 13 und 14 zu erwarten. Es kommt aber zu einem Perspektivenwechsel, denn im Mittelpunkt stehen andere Hauptakteure. Ein Haufen Flüchtlinge wartet im Freien unter schlechten Bedingungen auf ihre vorübergehende Unterbringung in einer Auffangstelle. Unter den Geflüchteten befinden sich Vertreter aller Alterskategorien. Sie fliehen ins Ziel- oder Transferland mit ihrem gesamten Hab und Gut, sodass sie wahrscheinlich nichts anderes als das, was auf dem Bild dargestellt wird, besitzen. Am traurigen Schicksal dieser Menschen wird jedoch in den begleitenden Texten wenig Anteil genommen, es wird zugunsten des Aufbaus und der Stärkung des Feindbilds ${ }^{4}$ eher bagatellisiert. Dementsprechend wird der Blick des Betrachters auf die negative Wahrnehmung gelenkt, sodass eher negative Emotionen statt des erwarteten Mitleids erregt werden.

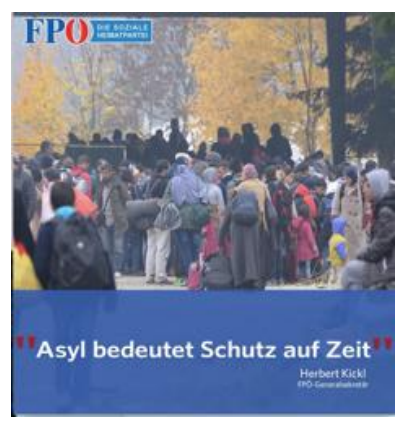

Abb. 13

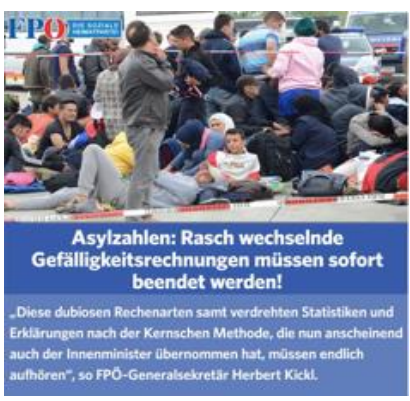

Abb. 14

4 Zur Instrumentalisierung des Feindbildes im Populismus vgl. z. B. Priester, „Wesensmerkmale des Populismus." 
Eine sehr ähnliche Situation liegt in Abbildung 15 vor. Der auf dem Boden sitzende Mann mit seinen auf den Kopf geschlagenen Händen erweckt den Eindruck, dass er etwas Trauriges, Unangenehmes erlebt hat und deshalb nun in Schmerz, Leid, Hoffnungslosigkeit und Verzweiflung versunken ist. Genauso gut aber kann diese Körperhaltung auch Faulenzerei, Nichtstun, Langeweile reflektieren. Aus der ambivalenten Interpretierbarkeit des Plakats ergeben sich Folgen in Bezug auf die Auslösung von Emotionen beim Betrachter, der mit dem Mann auf dem Plakat entweder mitfühlt oder sich über ihn empört.

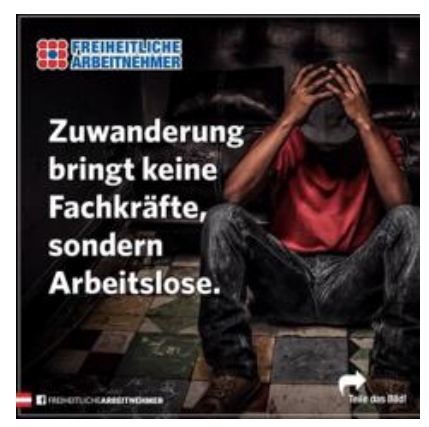

Abb. 15

Während die vorigen Beispiele auf das Gefühl des Mitleids als primäre Reaktion bei ihrer Wahrnehmung zurückzuführen sind, zeigt sich in Bezug auf die folgenden Bilder das Gefühl der Gefahr als emotionale Ausgangshaltung. Die Abbildungen 16-18 weisen auf die Notwendigkeit hin, die Grenzen des eigenen Landes bewachen zu müssen.

Auf dem Abb. 16 wird die Grenze durch den Stacheldraht geschützt. Ihre Überschreitung bringt schmerzhafte Folgen mit sich, dies aber für beide Seiten. In Abbildung 17 sorgt die Polizei für Ordnung an der Grenze. Wo auch immer die Polizei ist, erweckt das den Eindruck, dass etwas Schlimmes passiert ist oder etwas Gefährliches eintreten kann. Hand in Hand mit dem Gefühl der Bedrohung gehen Unsicherheit und Angst. Im Vordergrund von Abbildung 18 steht ein Mann, dessen Gesicht bis auf die Augen verhüllt ist. Solche Personen erwecken Misstrauen, Unsicherheit und Angst. Im Hintergrund ragen dichte Wälder und Berge der österreichischen Landschaft auf, um das Gefühl zu erwecken, dass sie nicht überstiegen werden dürfen. Die Dominante in diesem Bild sind jedoch die roten Blutflecken. Sie befinden sich überwiegend auf dem maskierten Mann, nichtsdestotrotz beflecken sie auch die Landschaft und die Berge. Indirekt wird damit auf den Täter eines potenziellen blutigen Massakers und dessen Zielgebiet hingewiesen. 


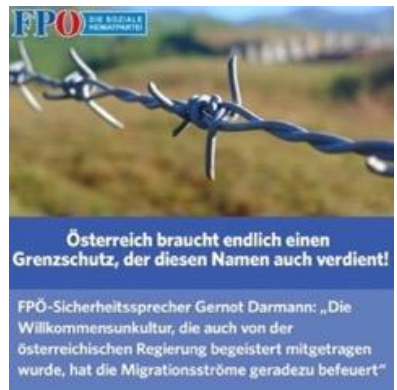

Abb. 16

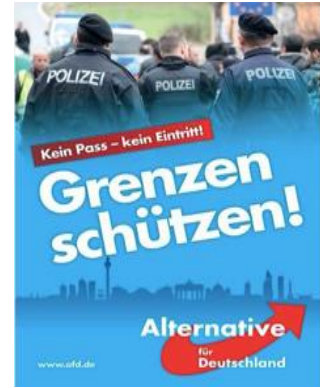

Abb. 17

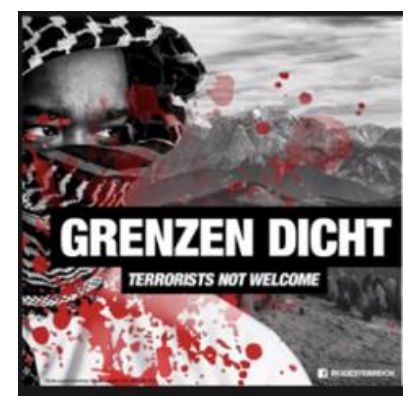

Abb. 18

Eine durchdachte visuelle Gestaltung demonstriert Abbildung 19. Auf einem rot-weißen Schlagbaum sitzen Geier, die sich ihrer „Beute“ nähern. Als Geier können Migranten und als Beute beispielsweise das soziale System des Ziellandes verstanden werden. Die angebliche Ausnutzung des sozialen Systems durch Migranten wird immer mit starker Empörung und Wut empfunden.

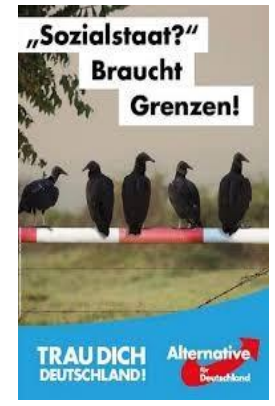

Abb. 19

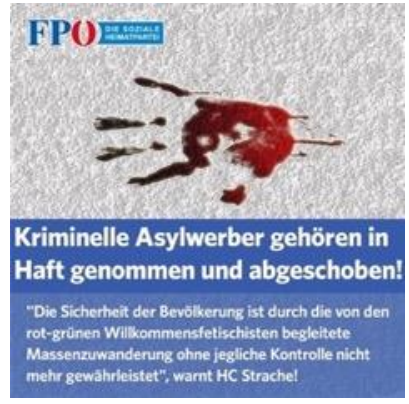

Abb. 20

Die ausgestreckte rote Hand auf dem Boden (Abb. 20) lässt zwei verschiedene Interpretationen zu: Erstens deutet sie auf ein blutiges Ereignis hin (Einbruch, Prügelei, Totschlag o. Ä.), zweitens weist sie als eine Art 
Stopp-Zeichen auf das Bedürfnis hin, diese Ereignisse zu verhindern. Das Thema Kriminalität wird auch auf in den Abbildungen 21-24 aufgegriffen.

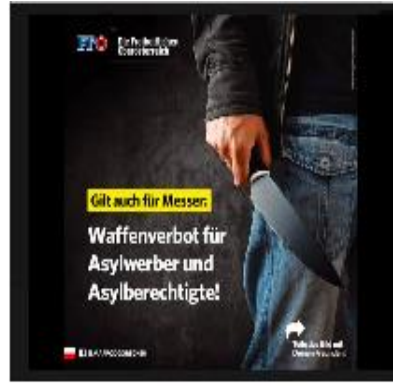

Abb. 21

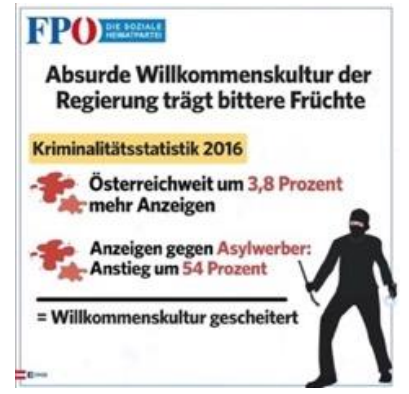

Abb. 22

In Abbildung 21 liegt die Konzentration auf einem langen Messer in der Hand. Das Messer wird als Waffe dargestellt, dementsprechend evoziert das Bild Gewalttaten und Menschenverletzungen. Abbildungen 22-24 demonstrieren wieder Einbruchssituationen mit Brecheisen. Im letzten Fall ist sogar die Eigentümerin anwesend, die verängstigt die Hand vor den Mund hält. Alle dargestellten Situationen erregen Besorgnisse und Ängste, denn sie können jede Person ohne Unterschied betreffen.

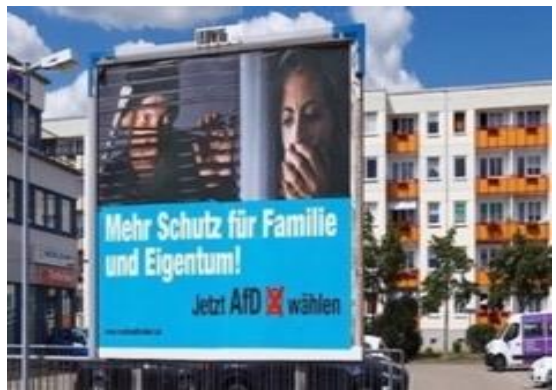

Abb. 23

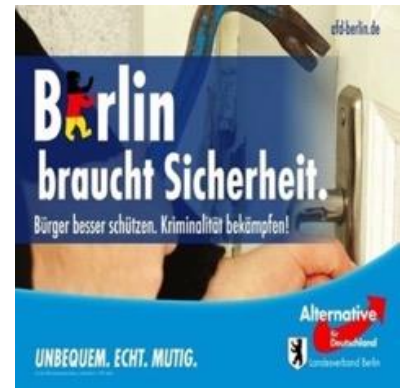

Abb. 24

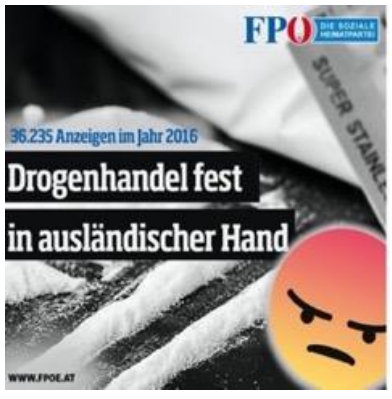

Abb. 25 
Auf die Kriminalität mit gefährlichen Folgen für die gesamte Gesellschaft, insbesondere aber die Jugend, die emotional instabiler ist, verweist ganz deutlich die schwarz-weiße Abbildung 25. Empörung und Missmut werden auch mit einem entsprechenden Emoticon zum Ausdruck gebracht. Parallel dazu lösen die Vorstellungen von potenzieller Drogensuchtgefahr auch Angst und Schauer aus.

\section{Effekte des Spiels mit Lust und Lachen}

Wie die bisherigen Beispiele und Analysen gezeigt haben, stellen negativ konnotierte Emotionen einen unbestreitbaren Bestandteil der sprachlichen Strategien im politischen Marketing des Rechtspopulismus dar und ziehen eine ganze Reihe von Effekten und Intentionen nach sich. Die bisherige Forschung war vor allem auf den negativen Pol der Emotionsskala fokussiert und hat kaum oder nur selten den Gemütsregungen im Umkreis des Lächelns und Lachens bzw. der Lust Aufmerksamkeit geschenkt. Im folgenden Teil dieses Beitrags wollen wir uns deshalb auch mit diesen vernachlässigten Aspekten näher auseinandersetzen.

Die wohl häufigste Darstellung auf politischen Plakaten, die natürlich auf das Erwecken von Sympathie beim Rezipienten abzielt, ist die eines lächelnden Kandidaten. Einerseits ähnelt politisches Marketing jeglichen anderen Formen der Produktwerbung und bezweckt es, beim Rezipienten eine positive Stimmung zu stimulieren. Andererseits muss das Lächeln jedoch gezähmt und in Grenzen gehalten werden und es sollte normalerweise nie oder nur selten in Lachen übergehen, da es sonst den politischen Repräsentanten unseriös und leichtsinnig erscheinen lassen könnte. ${ }^{5}$ Die komparative Analyse der Porträts von Politikerinnen und Politikern im Rahmen der untersuchten Datenbank ergab interessante Feststellungen. Die Vertreter der deutschen, slowakischen und tschechischen rechtspopulistischen Parteien sind in der Regel mit einer ernsten oder nur leicht lächelnden Miene dargestellt, dagegen findet man bei der österreichischen Partei FPÖ mehr Plakate mit einem breiten Lächeln bzw. einem lachenden Gesichtsausdruck der abgebildeten Person. Beispielhaft ist dafür insbesondere H. C. Strache, bei dem das Lachen auf das Idealbild eines charismatischen Führers zurückzuführen ist. ${ }^{6}$

5 Auch Pawlitschko und Pflüger verweisen darauf, dass man im Erwachsenenalter dazu neigt, das Lachen als unseriös zu empfinden. Vgl. Sven Pawlitschko, Markus Pflüger, „Gute Stimmung verkauft!“, in „Kauf mich!“ - Kommunikation: Verführen, verzaubern, verkaufen - ein So-geht's-Buch, hrsg. v. Stefan Gottschling (SGV Verlag, Augsburg 2014), 225.

6 Zum Merkmal des charismatischen Führers vgl. z. B. Decker, Frank, und Lewandowsky, Marcel. Rechtspopulismus: Erscheinungsformen, Ursachen und Gegenstrategien (Bonn: BPB, 
Demgegenüber sind die zum Extremismus neigenden politischen Figuren wie etwa Frauke Petri, Marine Le Pen oder M. Kotleba fast vorwiegend mit ernstem Gesicht dargestellt, was ihnen wahrscheinlich mehr Glaubwürdigkeit verleihen soll. ${ }^{7}$

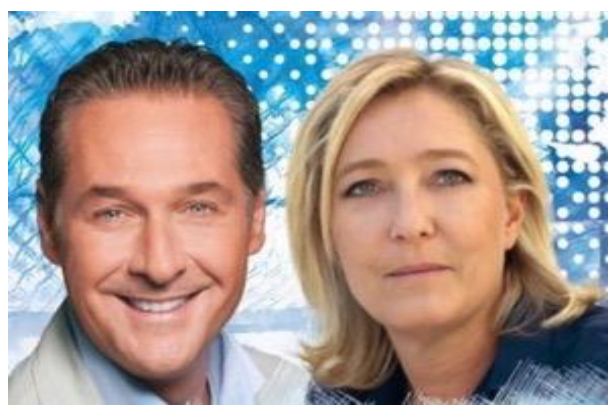

Abb. 26

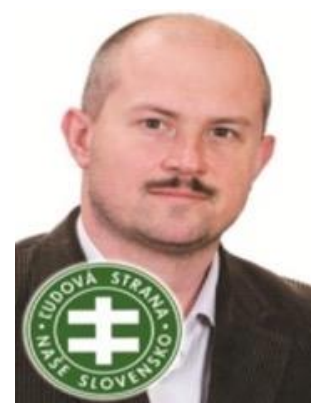

Abb. 27

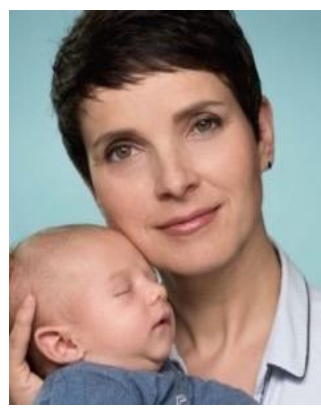

Abb. 28

Der charismatische Führer evoziert primär das Konzept des sogenannten Herzlandes, im Rahmen dessen das Gemeinschaftsgefühl und die Emotionen der Geborgenheit und Vertrautheit im Vordergrund stehen. ${ }^{8}$

2017): 25-27, https://www.bpb.de/politik/extremismus/rechtspopulismus/240089/ rechts-populismuserscheinungsformen-ursachen-und-gegenstrategien.

7 Die Facebook-Seiten von Frauke Petri zeigen im Unterschied zu den anderen Kandidaten auch Variationen des Porträts mit ernstem und lachendem Gesichtsausdruck. Vgl. Frauke Petri, Facebook, February 29, 2020, https://www.facebook.com/Dr.Frauke.Petry/.

8 Zum Konzept des Herzlandes vgl. z. B. Paul Taggart, Populism (Buckingham: Open University Press, PA 2000); Ján Demčišák, Simona Fraštíková, „The Analysis of Cultural and Visual Symbols in the Political Campaigns of the Right-Wing Populism in German Speaking Countries", in Reclaiming the future: official conference proceedings: official conference proceedings (Sakae: International Academic Forum, 2019), 71-88. 


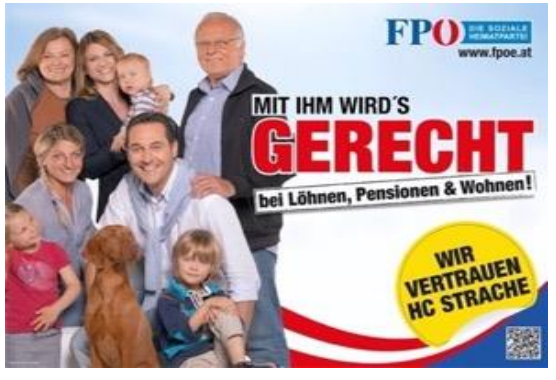

Abb. 29

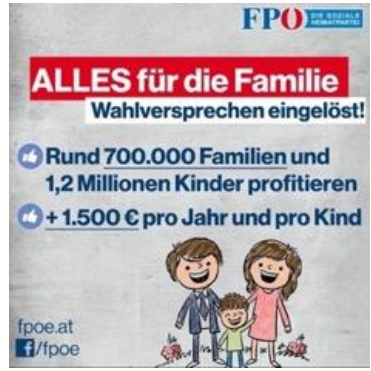

Abb. 30

In diesem Zusammenhang wird das Lachen zum Bestandteil einer sozialen Komponente, die der Anführer mit seinen Sympathisanten teilt. Zu lachen haben dabei allerdings nur diejenigen, deren Wohl durch das Handeln des Anführers oder seiner Formation gewährleistet wird. Nicht selten stehen im Fokus sensible Gruppen wie Kinder oder Rentner (ähnlich wie in den Beispielen, in denen es um Hervorrufung von Mitleid geht). Durch positive Einstimmung werden die Wähler konsolidiert und ihre Reaktion als Bestätigung der Richtigkeit der betriebenen Politik interpretiert. In diesem Sinne werden zufriedene „Kunden“ als Marketingmittel eingesetzt.

Lachende und glückliche Bevölkerungsgruppen können jedoch auch zum Auslöser für negative Emotionen werden, wie die beiden nächsten Beispiele zeigen.

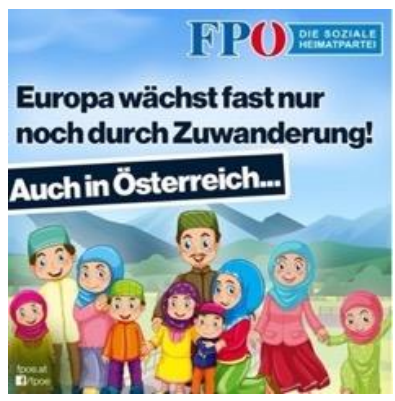

Abb. 31

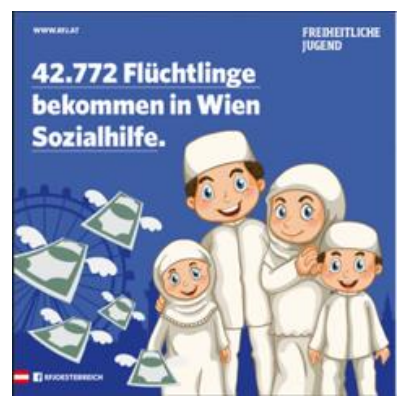

Abb. 32

Das Lachen des vermeintlichen Feindes soll in diesem Fall provokativ wirken und andere emotionale Effekte hervorrufen - gezielt wird insbesondere auf Emotionen wie Neid oder Angst. Unabhängig davon, ob es sich um einen negativen Impuls (Ausländer als Verbrecher und Kriminelle (vgl. Abb. $18,20,21,25$ ) oder um ein normalerweise positiv wahrzunehmendes Lachen handelt, wird gleichermaßen das Denken in Feindbildern angeregt. ${ }^{9} \mathrm{Bei}$

9 Zum Denken in Feindbildern vgl. z. B. Priester, „Wesensmerkmale des Populismus.“ 
einem inszenierten Feind wird Lachen als Gelächter und Auslachen des Rezipienten interpretiert. Die eigene Scham und das Gefühl lächerlich zu wirken oder dumm dazustehen verwandeln sich in Irritation und Hass und der zufriedene Bürger wird zum Wutbürger.

Je nach Bedarf ist Lachen also sowohl positiv als auch negativ konnotiert und kann ebenso vereinigenden wie auch differenzierenden Charakter haben. Die Rezipienten identifizieren sich entweder mit den Lachenden oder aber gehen auf Distanz. Dieser Freund/Feind-Schematismus ist auch bei der Darstellung der politischen Opponenten zu beobachten. So wird zum Beispiel Angela Merkel zumeist mit verbissener Miene dargestellt.

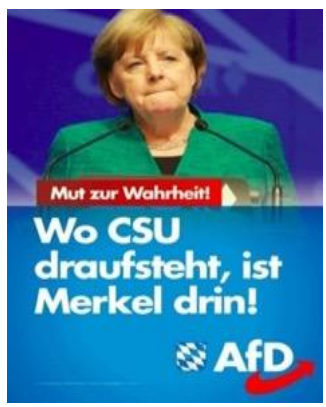

Abb. 33

Während in den früheren Beispielen die Neutralität des Ausdrucks und die Vermeidung von Emotionen mit Seriosität und Glaubwürdigkeit assoziiert werden sollten, führt die Absenz des Lächelns bzw. die verzerrte Mimik bei dem Gegner bewusst zur Stärkung des Moments der Antipathie.

In anderen Fällen dagegen wird der politische Gegenspieler direkt zum Gegenstand des Lachens. Die analysierte Bilderdatenbank enthält viele Belege für solche satirische Darstellungen, meistens in Form einer personalen Individualkarikatur.

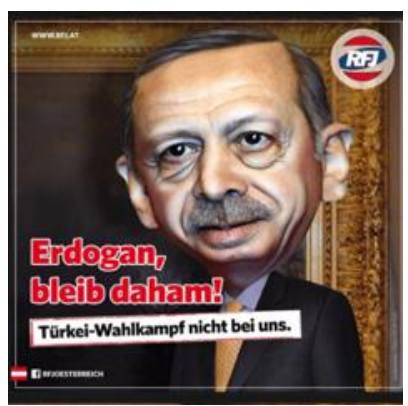

Abb. 34

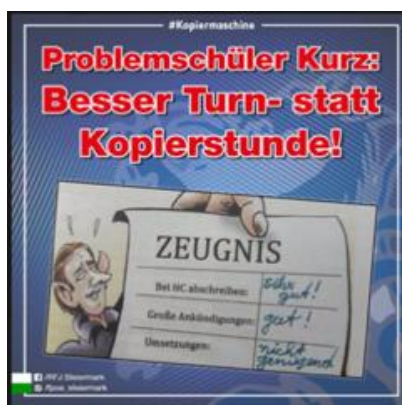

Abb. 35 


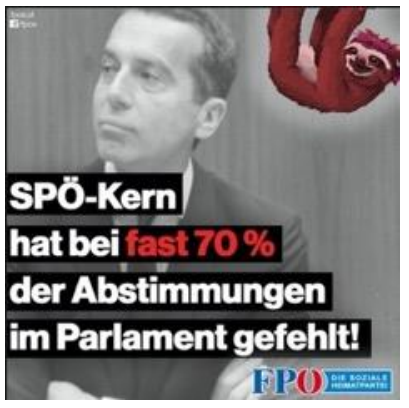

Abb. 36

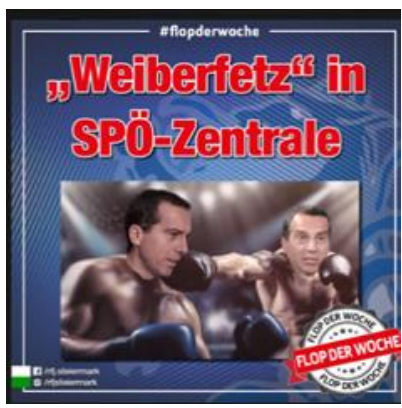

Abb. 38

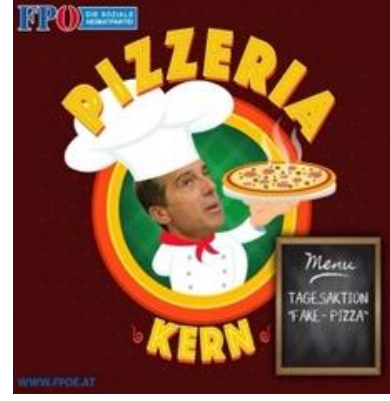

Abb. 37

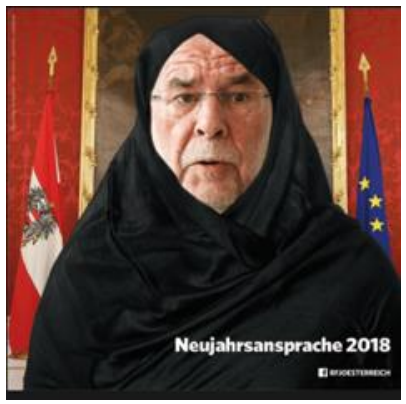

Abb. 39

Bei Abb. 34 wird das klassische stilistische Mittel der Karikatur - die überproportionale Darstellung eines Körperteils (hier des Kopfes) - eingesetzt, was an sich noch keine eigentliche Komik erzeugt. Diese entsteht teilweise durch die Korrelation zwischen Bild und Text und die Verwendung eines kinderreimähnlichen Slogans. Bei den anderen Beispielen versucht man dagegen die abgebildeten Personen durch den Kakao zu ziehen, indem ihnen verschiedene Laster und Untugenden zugeschrieben werden, wie Dummheit, Inkompetenz (Abb. 35), Faulheit (Abb. 36), Verlogenheit (Abb. 37) oder Streitsucht (Abb. 38). Die Zuschreibungen kommen dabei im Rahmen eines Vergleichs (Faultier, zankende Frauen) oder einer Maskerade (Problemschüler, Fake-News-Bäcker, Boxer, Muslimin) zustande und verfolgen das Ziel, den Konkurrenten lächerlich zu machen. In der patriarchalisch strukturierten und männlich konnotierten Politik wird auch von der Travestie als einer speziellen Form der Maskerade Gebrauch gemacht. ${ }^{10}$ Männer

10 Rekurriert wird auf die Begriffsbedeutung im Sinne des theatralischen und performativen Kleidertauschs, obwohl die in diesem Zusammenhang verwendete Technik der Verspottung auch Überschneidungen mit der literaturwissenschaftlichen und stilistischen Auffassung dieses Begriffs aufweist. Vgl. Metzler Literatur Lexikon, hrsg. v. Dieter Burdorf, and Christoph Fasbender, Burkhard Moenninghoff (Stuttgart: Metzler, 2010), 780. 
werden entweder mit „Weibern“ verglichen (Abb. 38) oder direkt als Frauen stilisiert (Abb. 39), wobei die Effemination zur Herabwürdigung ihrer Männlichkeit und Männerwürde führt. Auf der einen Seite wird mit Klischees und Stereotypen gearbeitet, auf der anderen Seite werden diese bewusst oder unbewusst untermauert und weiterverbreitet. Beim letzten Beispiel geht es aber nicht nur um die Verweiblichung des Mannes, die lächerlich wirken soll, sondern darüber hinaus wird durch das muslimische Gewand auch der religiöse Blickwinkel satirisch entstellt. Das Ineinandergreifen dieser beiden unterschiedlichen Ebenen und ihr Zusammenwirken, aus dem der komische Effekt entsteht, entsprechen dem, was Freud in seinen Überlegungen bezüglich der Technik des Witzes mit dem Begriff Verdichtung bzw. Verdichtungswitz bezeichnet.11

In der nächsten Abbildung (40) wird die Grundstruktur und Idee von Abbildung 38 übernommen und variiert.

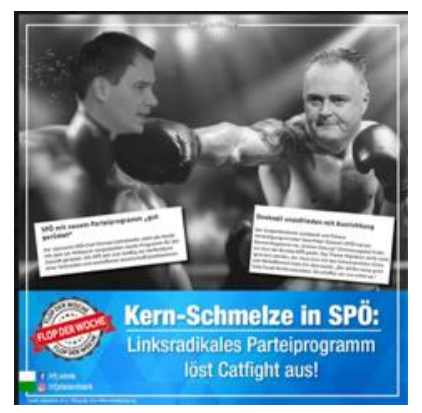

Abb. 40

Während bei den Wortwitzen diverse Variationen und sogar eine Art Serialität üblich sind, handelt es sich bei den von uns untersuchten Plakaten eher um eine Ausnahme. Wiederaufgegriffen wird das Motiv der Streitsucht und der Effemination durch die Anspielung auf "Catfight". Auf der Wortebene wird wortspielerisch mit dem Namen von Christian Kern umgegangen, obwohl der Parteichef der SPÖ selbst nicht im Bild vorkommt. Als „Kern-Schmelze“ wird die destabilisierende Reaktion auf das neue Parteiprogramm innerhalb der SPÖ bezeichnet, über die die FPÖ-Sympathisanten lachen können. In der Freudschen Typologie kann man von einem sogenannten Zerteilungswitz sprechen, der bei der Zerlegung eines Wortes in mehrere Teile entsteht. ${ }^{12}$

11 Vgl. Sigmund Freud, Der Witz und seine Beziehung zum Unbewussten (Leipzig und Wien: Franz Deuticke, 1905), 10-12.

12 Vgl. Freud, Der Witz und seine Beziehung zum Unbewussten, 21. 
Einen Grund zum Lachen bzw. zur Freude bietet dem sich mit der FPÖ identifizierenden Rezipienten jedoch allein die Tatsache, dass die Probleme in der gegnerischen Partei zum Erfolg der eigenen beitragen können. Im Prinzip geht es um die Position des lachenden Dritten im Sinne der Redewendung „Wenn sich zwei streiten, freut sich der Dritte“. Das Motiv der Schadenfreude spielt deshalb eine wichtige Rolle auch bei weiteren Bildern, wo das Scheitern der Gegner thematisiert wird.

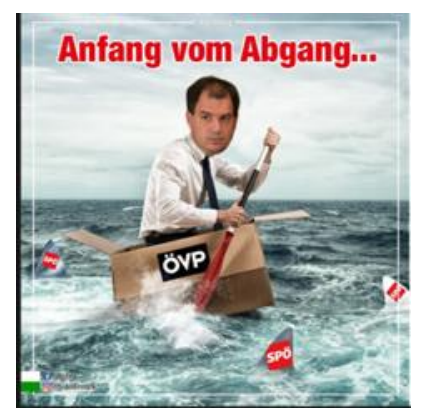

Abb. 41

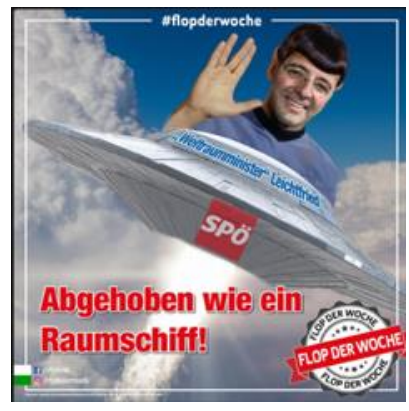

Abb. 42

Auch hier handelt es sich primär um die personale Individualkarikatur, auf den Bildern 41 und 42 treten jedoch auch unpersönliche politische Subjekte auf, die satirisch als abfliegendes Raumschiff, sinkendes Kartonboot oder als Haie abgebildet werden und genau wie die vorigen Beispiele auf den Konkurrenzkampf oder den Misserfolg des Gegners hinweisen.

Ähnlich ist es auch bei den nachstehenden Bildern, die aufgrund der abstrakten Darstellungen eigentlich dem Typ der Sachkarikatur bzw. der Typenkarikatur zuzuordnen sind und wiederum im Zeichen der politischen Rivalität stehen.

In Abbildung 43 werden die Schwierigkeiten in der Regierungskoalition der SPÖ und ÖVP verspottet.

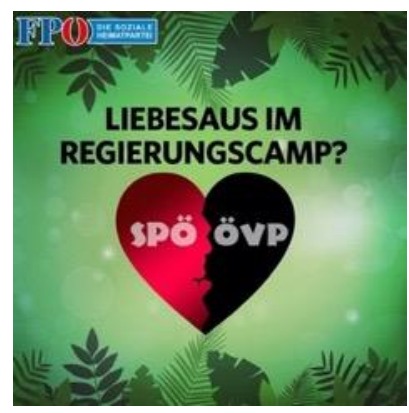

Abb. 43 
Die Symbolik des Herzens, die normalerweise mit positiven Konnotationen besetzt ist, wird ironisch gebrochen, sodass das schwarz-rote Herz auf die Disharmonie einer Liebesbeziehung verweist. Um die Satire der Darstellung entschlüsseln zu können, wird vom Rezipienten erwartet, dass er die Anspielung auf den medialen Kontext versteht. Der grüne, florale Hintergrund und der Begriff „Regierungscamp“ verweisen auf die Fernsehshow „Dschungelcamp“, in der verschiedene, oft zweitklassige Prominente darum kämpfen, wer es am längsten in dem Camp aushält und die größte Popularität gewinnt. Die Konkurrenten werden somit als aufmerksamkeitssüchtige Stars entlarvt.

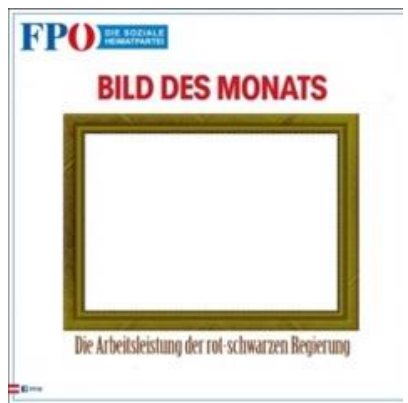

Abb. 44

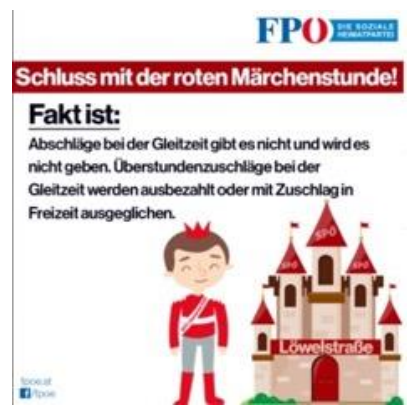

Abb. 45

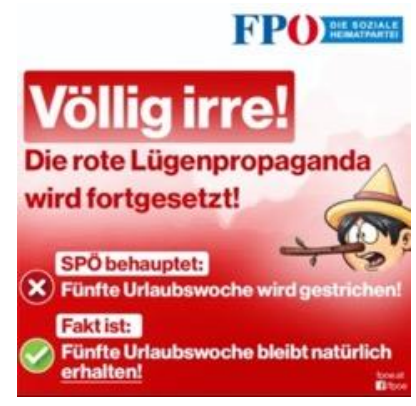

Abb. 46

Im Zentrum der anderen Darstellungen stehen, wie es schon vorher bei den personalen Karikaturen der Fall war, die Laster und Schwächen der Gegner. Kritisiert wird ihre Unfähigkeit und mangelnde Arbeitsleistung (Abb. 44) oder sie werden als Märchenerzähler und Lügner bezeichnet (Abb. 45 und 46). Die Figuren des realitätsfernen Märchenprinzen oder des unglaubwürdigen Pinocchio können in diesem Fall als Typenkarikaturen klassifiziert werden.

Auch das Abb. 47 bedient sich einer typologischen Darstellung, die Tradition (Paar in Tracht) und Migration (bedrohliche Verbrecher) in Gegensatz 
stellt und gegeneinander ausspielt. Der Kontrast wird zusätzlich auch farblich (bunt versus grau) und mimisch (Fröhlichkeit versus Grimm und Arglist) verdeutlicht. Im Gegensatz zur Zeichnung der Migranten im Kontext negativer Emotionen - mit dem erwarteten Effekt der Furcht, Bedrohung oder Wut (vgl. vorheriges Kapitel) - soll hier die Gefahr eher verharmlost werden. Deswegen wirken die karikierten Migranten eher lächerlich wie unfähige Lumpen und Gauner, die leicht zu schlagen sind.

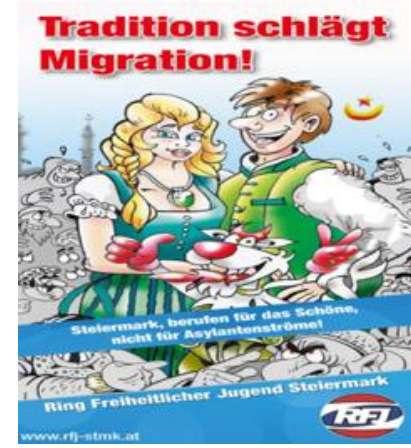

Abb. 47

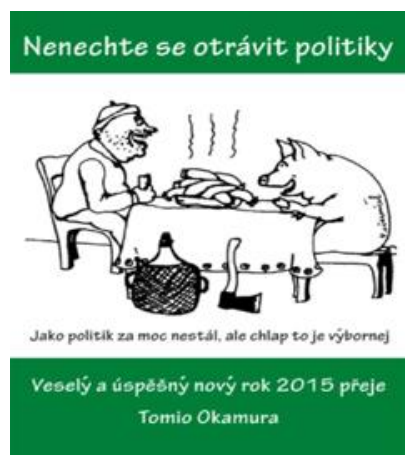

Abb. 48

Teilweise schwankend zwischen Sach- und Typenkarikatur ist die nächste Abbildung (48), ein Plakat der tschechischen Partei „Úsvit“. Im Mittelpunkt des Neujahrswunsches des Parteivorsitzenden Tomio Okamura steht ein Witz, der auf mehreren Ebenen wirkt. Am Tisch sitzen gemeinsam ein Mann und eine Sau und verspeisen Blutwürste. Der Zeichnung ist ein Kommentar beigefügt, der besagt: „Als Politiker taugte er zu nichts, aber als Kerl ist er ausgezeichnet." So formuliert eröffnet der Satz mehrere Leerstellen, die der Rezipient selbst ergänzen und die Szene individuell interpretieren kann, denn es ist nicht eindeutig, ob es sich um die Aussage des Mannes, des Schweines oder eines außenstehenden Betrachters handelt. In diesem Sinne könnte mit dem Kerl entweder der abgebildete und ein bisschen verwahrlost aussehende Mann oder ein anderer ehemaliger Politiker, von dem die Figuren reden, gemeint sein. Es kann sogar sein, dass es sich um schwarzen Humor handelt und dass aus dem Politiker nun die Blutwürste sind. Als Indiz dafür dient das Beil. Die kannibalische Komponente ist eindeutig präsent, entweder verzehrt der Mann einen Politiker oder aber ist es das Schwein, das die Schweineblutwurst frisst. Je nachdem, woraus die Blutwurst besteht, stellt das Beil für einen der Tischgenossen eine mögliche Gefahr dar. Mit satirischen und ironischen Mitteln wird das Wesen der Politik und der Politiker karikiert. Angeprangert werden Unehrlichkeit und Hinterlist, die zugespitzt mit Kannibalismus assoziiert werden. Den Politikern sei 
nicht zu vertrauen, denn sie sind verlogen und unberechenbar wie Alkoholiker (Indiz Weingefäß links neben dem Beil). Paradoxerweise wird dieser Witz von einem Politiker veröffentlicht. Als seine Intention kann man deshalb die Abgrenzung von einem solchen Politikstil vermuten. Um dies zu verdeutlichen, ergänzt Okamura das Bild mit einem eigenen Wortwitz, der die Doppeldeutigkeit der Karikatur aufgreift, was damit zusammenhängt, dass sich das tschechische Wort „otrávit“ unterschiedlich deuten lässt. Im übertragenen Sinn heißt der Satz übersetzt: „Lassen Sie sich nicht von den Politikern verärgern“, wortwörtlich wird jedoch gemeint: „Lassen Sie sich nicht von den Politikern vergiften“, was direkt auf die Szene mit dem Essen anspielt. In den Augen des Rezipienten werden Politiker als Menschen betrachtet, die einen belügen, betrügen, ausnutzen und sogar umbringen können.

Eine ähnliche Idee liegt auch der nächsten Karikatur (Abb. 49) ab, die ebenso von der Partei „Úsvit“ stammt. Für das Jahr 2014 wünscht sie ihren Wählern eine „echte“ bzw. wahre „Demokratie“, wobei hier auf witzige Weise Tod und Freiheit einander gegenübergestellt werden.

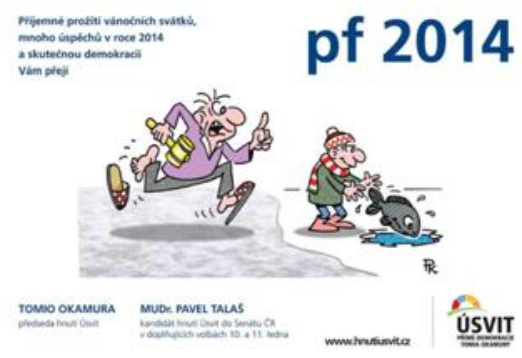

Abb. 49

Mit der Hervorhebung von Kontrasten und Unterschieden arbeitet auch ein winterlicher Weihnachtsgruß der tschechischen SPD aus dem Jahr 2017 (Abb. 50). Für den Witz soll in diesem Fall der kinderreimartige Text wirken. Das Gedicht kommentiert die kulturelle Tradition des Beschenkens zu Weihnachten, indem es uns daran erinnert, dass bei uns die Weihnachtsgeschenke vom Christkind und nicht von Ali-Baba aus Tausendundeine Nacht kommen. Es wird zugleich subsumiert, dass man uns diesen Gedanken in unsere Köpfe einpflanzen möchte. Der Witz verfälscht mit Absicht die Wahrnehmung der anderen Tradition und Kultur und schlägt somit in ein Gefühl der kulturellen Bedrohung oder aber der Überlegenheit um.

Die Strategie des Konterkarierens einer fremden Kultur ist auch in $\mathrm{Zu}-$ sammenhang mit Abb. 51, einem Plakat der RFJ Steiermark, zu erkennen, auf dem man sich über die muslimischen Gebetsrituale lustig macht, indem man 
sie mit Turnübungen vergleicht und für eine Gesundheitsvorsorge hält. Durch die Emoticons wird dem Rezipienten zusätzlich die richtige Einstellung zu diesem Thema suggeriert.

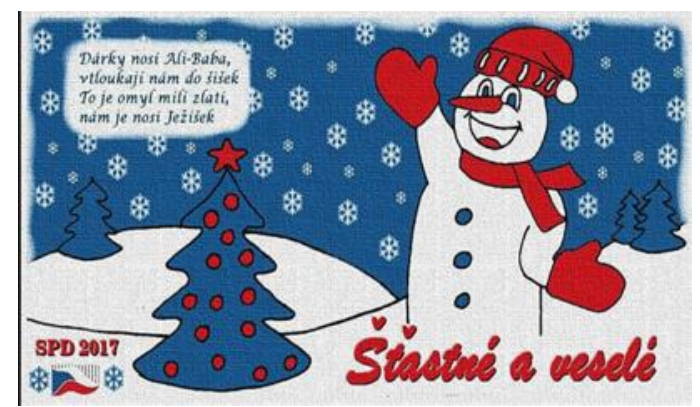

Abb. 50

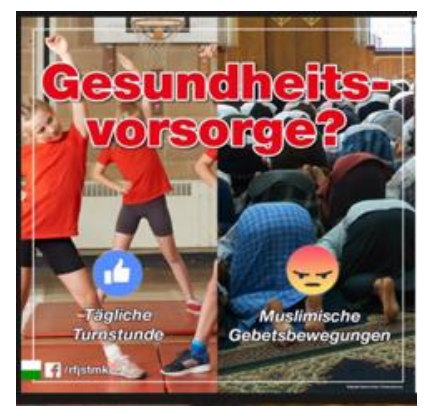

Abb. 51

Migranten und Ausländern wird nicht nur unterstellt, dass sie unsere kulturellen Werte unterlaufen, sondern auch, dass sie an dem sozialen System und Wohlstand schmarotzen wollen. Diese Sachverhalte werden nicht selten unter der Anwendung von Ironie kommuniziert, wie Abbildung 52 belegt.

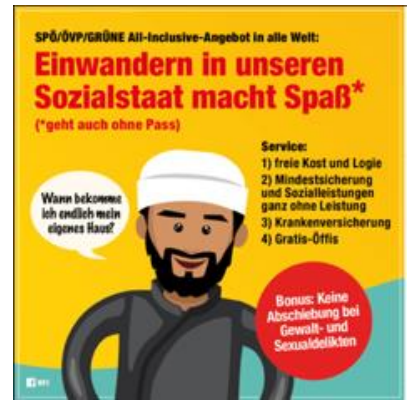

Abb. 52

Behauptet wird, dass Einwanderung Spaß macht, dass Gewalt und Sexualdelikte belohnt werden können oder dass die Situation der Flucht und Migration mit einem Urlaub mit All-Inclusive-Angebot zu vergleichen ist. Die Absicht ist jedoch nicht, den Rezipienten zum Lachen zu bringen, im Gegenteil, ähnlich wie bei den vorher analysierten Bildern mit lachenden Flüchtlingen (Abb. 31 und 32), sollen Irritation und Wut hervorgerufen werden.

Einen interessanten Sonderfall stellen die folgenden Beispiele der tschechischen politischen Subjekte Úsvit bzw. SPD dar. Sie zeigen nämlich, dass im Rahmen der politischen Kommunikation auch eine selbstironische Darstellung möglich ist. In Abbildung 53 stellt sich die Partei satirisch und selbstironisch als Reinigungsmittel dar, das konzentriert und unverdünnt 
gegen politischen Dreck und Korruption wirkt. Ein anderes Mal bereitet die Partei für ihre Wähler eine Variation des Spiels Mensch ärgere dich nicht (Abb. 54), bei dem es unterschiedliche Aktionsfelder gibt, die Bezug auf reale politische Akteure und die aktuelle Situation nehmen. Der Name des Spiels „Okamuras Häuschen" ist zugleich eine ironische Anspielung an den Wunsch der Partei, die Wähler im eigenen Häuschen zu haben. Das dritte Beispiel karikiert den Parteivorsitzenden der Partei zusammen mit Marine Le Pen als Rattenfänger, die Europa von unangenehmen „Ratten“ befreien.

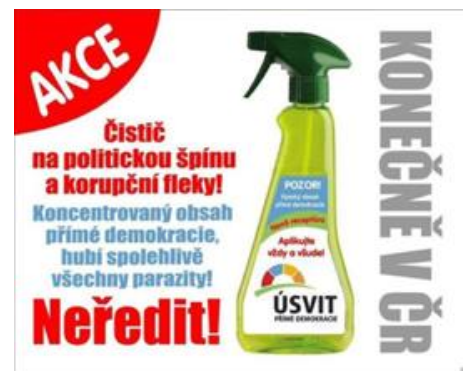

Abb. 53

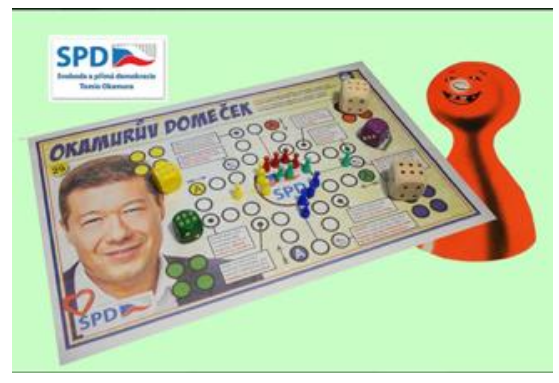

Abb. 54

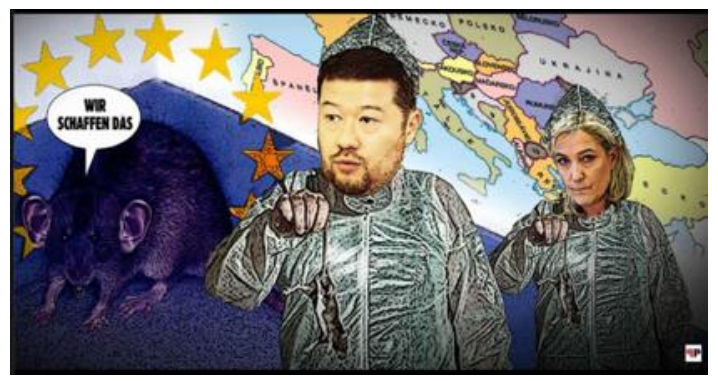

Abb. 55

Satire und Ironie richten sich im politischen Diskurs in der Regel gegen die politischen Gegner, oder die politischen Parteien und deren Repräsentanten werden zum Inhalt einer allgemeinen politischen Satire und Karikatur. Politische Plakate ziehen auch häufig die Kreativität der Bevölkerung nach sich und werden zur Zielscheibe von Witzen, Entstellungen oder künstlerischen Parodien. ${ }^{13}$ Insofern stellte die ironische Selbstdarstellung von Tomio Okamura eine Ausnahme dar. Die Intention aber ist, dass beim Rezipienten Sympathien für den humorvollen Politiker geweckt werden, was die

13 Vgl. z. B. die künstlerische Parodie der AfD-Plakate von Shahak Shapira, 29 Februar, 2020, https://www.bento.de/politik/afd-wahlplakate-zum-islam-shahak-shapira-verarschtdie-partei-a-00000000-0003-0001-0000-000001420889. 
Identifikation mit der Partei besser stärken kann als die bloße Vorstellung eines gemeinsamen Feindes.

\section{Nachspiel}

Bei der Wahrnehmung von Bildern mit politischen Inhalten entfalten sich Emotionen oft stufenweise; aus dem direkt im Moment der Betrachtung des Plakats individuell empfundenen Gefühl ergeben sich unter Einbeziehung der Bewertung des Abgebildeten oft weitere Emotionen und Effekte. Außerdem werden die Emotionen als unmittelbare Effekte der Wahrnehmung entweder im direkten Verhältnis zum Dargestellten empfunden (z. B. Angst vor Verbrechern) oder vermittelt und verschoben im Verhältnis zu einem Dritten (z. B. Mitgefühl mit den Rentnern verwandelt sich in Angst um sich selbst oder in Wut gegen die verantwortlichen politischen Parteien). Die primären emotionalen Reaktionen können also nicht immer und überall als endgültige emotionale Einstellung zum dargestellten Sachverhalt erfasst werden.

Für die emotionale Einstellung zu den abgebildeten Inhalten sind dabei zwei Ausgangsemotionen ausschlaggebend: Gefühl des Mitleids und Gefühl der Bedrohung. Ihre Wahrnehmung ist jedoch primär von der Wahl des dargestellten Inhalts oder Akteurs abhängig, entscheidend ist die Tatsache, ob sich der Rezipient mit diesen Sachverhalten oder Personen identifizieren kann. Die Identifikationsmöglichkeit ist jedoch auch für das Empfinden von Lust und Freude wesentlich. Die lachenden oder lächelnden Akteure bewirken beim Rezipienten einerseits positive Reaktionen und andererseits eher Irritation und Wut. Dabei gilt, dass das positiv konnotierte, insbesondere aufgrund der Identifikation mit dem Dargestellten entstandene Gefühl (Mitgefühl, Lust) häufig zur Auslösung von negativen Emotionen führt. Das negativ konnotierte Gefühl verwandelt sich in der Regel nicht in sein Gegenteil.

Die Prämissen des Einfühlungsvermögens und der potenziellen Identifikation lassen sich mit der Aktivierung eines Freund/Feind-Schematismus in Zusammenhang bringen. Dieses Schema basiert auf der Stratifizierung und Hierarchisierung von sozialen Strukturen. Bezogen auf die politische Realität geht es darum, dass man sich als Teil eines Kollektivs (einer Nation, einer Partei) wahrnimmt und infolge dessen alle Außenstehenden als potenzielle Gegner betrachtet. Gegen die Außenseiter werden vor allem negative Emotionen gehegt (Hass, Wut, Misstrauen, Angst um sich selbst). Diese entstehen entweder direkt (z. B. aufgrund einer Frustration) oder nachträglich als Folge der ursprünglichen Lustempfindung entstehen. 
Im Umkreis der Lust und des Humors geht es nur selten um das Erwecken von Sympathie auf der Grundlage von Lächeln oder Freundlichkeit. Sympathisch und positiv sollen vielmehr die Ziele und Erfolge der Partei wirken, die aber oft mit dem Empfinden von Mitleid oder mit Altruismus assoziiert werden, wie bereits beschreiben wurde. Als Anreger von Lust dominieren dagegen Hohn, Verspottung und Auslachen, die sich satirisch gegen den politischen Gegner richten. Das gemeinsame Lachen über den Feind wirkt identitätsstiftend. Der Mechanismus der komischen Wirkung lässt sich in diesem Kontext aber auch mit der sogenannten Überlegenheitstheorie erklären, die auf Platon und Aristoteles zurückgeht. ${ }^{14}$ Dieser Auffassung zufolge ist Komik eine abfällige Reaktion auf die Umwelt, wobei das Lachen dem Lachenden das Gefühl verleiht, dem Ausgelachten überlegen zu sein. Der Rezipient wird somit in die Position des Klügeren, des Wissenden bzw. eines moralisch besseren Menschen versetzt, was einerseits zu einer positiven Selbstwahrnehmung führt und anderseits eine Gemeinschaft mit den gemeinsam Lachenden und Gleichgesinnten erzeugt. Anders ist es jedoch im Falle der Selbstironie, wo dieser Erklärungsansatz ungenügend ist. Die Fähigkeit, über sich selbst zu lachen, bedeutet, teilweise eigene Schwächen anzuerkennen oder mindestens bereit zu sein, dem Auslachen oder Spott ausgeliefert zu werden. Dies lassen die populistischen Parteien in der Regel nicht zu und deswegen stellt der Gebrauch von Selbstironie eher eine Ausnahme dar. Jedoch auch in diesem Fall liegt das strategische Ziel darin, die Sympathie des Rezipienten für sich selbst zu gewinnen.

Mit dem Lustprinzip kann unter Umständen auch der Wirkungsmechanismus des Mitgefühls erklärt werden. Im Rahmen der These des sogenannten psychologischen Egoismus wird behauptet, dass die tatsächliche Motivation für altruistisches Handeln in unserem eigenen Egoismus zu suchen ist. ${ }^{15}$ Demzufolge kann Mitleid dem Rezipienten auch das Gefühl geben, dem sozial Schwächeren oder einem Opfer überlegen zu sein; damit verbunden kann einem das eigene Glück im Vergleich zum Unglück des anderen bewusst werden.

Wie sich aus unseren Analysen ergibt, sind die tatsächlichen Effekte der Affekte an sich ambivalent. Bei der Entstehung einer emotionalen Wirkung oder Wahrnehmung spielt eine wesentliche Rolle die eigentliche Identifikation oder Nicht-Identifikation des Betrachters mit den Darstellungsinhalten,

14 Vgl. Platons Philebos bzw. Poetik von Aristoteles. Platon, Philebos. In Platons Werke. Zweiten Teiles dritter Band, hrsg. v. Friedrich D. Schleiermacher (Berlin: Georg Reimer Verlag,1861), http://www.opera-platonis.de/Philebos.pdf; Aristoteles, Poetik, übers. und hrsg. v. Manfred Fuhrmann (Stuttgart: Philipp Reclam, 1982), 17.

15 Vgl. Franz Kutschera, Grundlagen der Ethik (Berlin: De Gruyter, 1999), 67-69. 
die auf die innere Veranlagung und Wertvorstellung des Rezipienten zurückgeht. Es zeigt sich aber auch etwas Anderes. Die Identifikation des Rezipienten mit dem Produzenten der kommunizierten Botschaft bzw. den dargestellten Sachverhalten kann zusätzlich durch eine bewusste Steuerung in Richtung Sympathie oder Antipathie, bei der die zustande kommende Gemütslage eine wesentliche Rolle spielt, entweder angeregt oder verhindert und verschoben werden. Es handelt sich um eine Strategie, die von populistischen politischen Subjekten gezielt und - was die politische Entwicklung unserer Zeit beweist - mit Erfolg verwendet wird. Die Demaskierung und Kenntnis von solchen manipulativen Techniken können uns hoffentlich helfen, der Manipulation des Rechtspopulismus und Extremismus entgegenzuwirken.

\section{References}

AfD-Plakate von Shahak Shapira, February 29, 2020. https://www.bento. de/politik/afd-wahlplakate-zum-islam-shahak-shapira-verarscht-diepartei-a-00000000-0003-0001-0000-000001420889.

Aristoteles. Poetik. Translated and edited by Manfred Fuhrmann. Stuttgart: Philipp Reclam, 1982.

Decker, Frank, and Marcel Lewandowsky. Rechtspopulismus: Erscheinungsformen, Ursachen und Gegenstrategien. Bonn: BPB, 2017. https://www. bpb.de/politik/extremismus/rechtspopulismus/240089/rechts-populismuserscheinungsformen-ursachen-und-gegenstrategien.

Demčišák, Ján, and Simona Fraštíková. "The Analysis of Cultural and Visual Symbols in the Political Campaigns of the Right-Wing Populism in German Speaking Countries." In Reclaiming the future: official conference proceeding: official conference proceedings, 71-88. Sakae: International Academic Forum, 2019.

Frauke Petri, Facebook, February 29, 2020. https://www.facebook.com/ Dr.Frauke.Petry/.

Freud, Sigmund. Der Witz und seine Beziehung zum Unbewussten. Leipzig und Wien: Franz Deuticke, 1905.

Kutschera, Franz. Grundlagen der Ethik. Berlin: De Gruyter, 1999.

Lexikon der Biologie [Stichwort:] “Weinen.” https://www.spektrum.de/lexikon/biologie/weinen/70503.

Metzler Literatur Lexikon, edited by Dieter Burdorf, and Christoph Fasbender, and Burkhard Moenninghoff. Stuttgart: Metzler, 2010.

Pawlitschko, Sven, and Markus Pflüger. Gute Stimmung verkauft! In "Kauf mich!" - Kommunikation: Verführen, verzaubern, verkaufen - ein So- 
geht's-Buch, edited by Stefan Gottschling, 217-230. Augsburg: SGV Verlag, 2014.

Platon, Philebos. In Platons Werke. Zweiten Teiles dritter Band, edited by Friedrich D. Schleiermacher. Berlin: Georg Reimer Verlag, 1861. http://www.opera-platonis.de/Philebos.pdf.

Priester, Karin. "Das Syndrom des Populismus." Dossier: Rechtspopulismus (Bonn: BPB, 2017): 7-12. www.bpb.de/system/files/pdf_pdflib/pdflib241384.pdf.

Priester, Karin. "Wesensmerkmale des Populismus." Aus Politik und Zeitgeschichte 62 (Bonn: BPB, 2012): 3-9.

Spier, Tim. "Populismus und Modernisierung." In Populismus, edited by Frank Decker, 33-58. Wiesbaden: VS Verlag für Sozialwissenschaften, 2006.

Taggart, Paul. Populism. Buckingham: Open University Press, (PA), 2000.

\section{Bilderverzeichnis}

Abb. 1: https://www.facebook.com/fpoe/photos/a.170548806419730/ 779604772180794/?type=3\&theater, March 6, 2019.

Abb. 2: https://www.afdbayern.de/wahlen-2018/themenplakate/, March 6, 2019.

Abb. 3: https://www.afdbayern.de/wahlen-2018/themenplakate/, March 6, 2019.

Abb. 4: https://www.facebook.com/865985613586144/photos/a.866075096910529/ 987570554760982/?type=3\&theater, March 6, 2019.

Abb. 5: https://www.t-online.de/nachrichten/deutschland/parteien/id_77174628/verdacht-auf-illegale-parteispende-bei-der-afd.html, March 6, 2019.

Abb. 6: https://www.facebook.com/fpoe/photos/a.170548806419730/1179288 575545743/?type=3\&theater\%20(10.\%2003.\%202019), March 6, 2019.

Abb. 7: https://www.facebook.com/fpoe/photos/a.170548806419730/ 963691877105415/?type=3\&theater, March 6, 2019.

Abb. 8: https://www.facebook.com/865985613586144/photos/a.866075096910529/ $866821590169213 /$ ?type=3\&theater, March 6, 2019.

Abb. 9: https://www.facebook.com/865985613586144/photos/a.866189970232375/ 980401555477882/?type=3\&theater, March 6, 2019.

Abb. 10: https://www.facebook.com/fpoe/photos/a.170548806419730/ 749634141844524/?type=3\&theater, March 6, 2019.

Abb. 11: http://www.gemeinsamfuerdeutschland.de/index.php/landtagswahl-2018, March 6, 2019.

Abb. 12: https://www.facebook.com/fpoe/photos/a.170548806419730/ 1240436826097584/?type=3\&theater, March 10, 2019.

Abb. 13: https://www.facebook.com/fpoe/photos/a.170548806419730/ 697924360348836/?type=3\&theater, March 6, 2019.

Abb. 14: https://www.facebook.com/fpoe/photos/a.170548806419730/ 708142772660328/?type=3\&theater, March 6, 2019.

Abb. 15: https://www.facebook.com/865985613586144/photos/a.866189970232375/ 990453321139372/?type=3\&theater, March 6, 2019. 
Abb. 16: https://www.facebook.com/fpoe/photos/a.170548806419730/ 687355431405729/?type=3\&theater, March 6, 2019.

Abb. 17: http://www.afd-pi.de/z_galerie/, March 6, 2019.

Abb. 18: https://www.facebook.com/FJOesterreich/photos/a.307509049850/ 10154881401329851/?type=3\&theater, March 6, 2019.

Abb. 19: https://www.horizont.net/galerien/Populistisch-nicht-populaer-3255, March 6, 2019.

Abb. 20: https://www.facebook.com/fpoe/photos/a.170548806419730/ 676479892493283/?type=3\&theater, March 6, 2019.

Abb. 21: https://www.facebook.com/865985613586144/photos/a.866075096910529/ 1000307843487253/?type=3\&theater, March 6, 2019.

Abb. 22: https://www.facebook.com/fpoe/photos/a.170548806419730/ 839102109564393/?type=3\&theater, March 6, 2019.

Abb. 23: http://afd.berlin/wahlplakate-2016/, March 6, 2019.

Abb. 24: https://www.faz.net/aktuell/politik/wahl-in-mecklenburg-vorpommern/afd-erhaelt-wahlunterstuetzung-von-verein-in-mecklenburg-vorpommern-14398142.html, March 6, 2019.

Abb. 25: https://www.facebook.com/fpoe/photos/a.170548806419730/ 920176694790267/?type=3\&theater, March 6, 2019.

Abb. 26: https://www.facebook.com/fpoe/photos/a.170548806419730/ 692165080924764/?type=3\&theater, March 6, 2019.

Abb. 27: http://www.naseslovensko.net/wp-content/uploads/2016/02/nase-slovenskofebruar-2016.pdf, April 1, 2019.

Abb. 28: https://www.designtagebuch.de/die-plakate-zur-bundestagswahl-2017/afd-plakat-petry/, January 28, 2019.

Abb. 29: https://www.facebook.com/fpoe/photos/a.170548806419730/ 243216889152921/?type=3\&theater, March 12, 2019.

Abb. 30: https://www.facebook.com/fpoe/photos/a.170548806419730/ 1005911869550082/?type=3\&theater, March 6, 2019.

Abb. 31: https://www.facebook.com/fpoe/photos/a.170548806419730/ 1102438889897379/?type=3\&theater, March 10, 2019.

Abb. 32: https://www.facebook.com/FJOesterreich/photos/a.307509049850/ 10155485716044851/?type=3\&theater, January 1, 2019.

Abb. 33: ttps://www.afdbayern.de/wahlen-2018/themenplakate/, December 7, 2018.

Abb. 34: https://www.facebook.com/rfj.stmk/photos/a.217993564882394/ 1684770948204641/?type=3\&theater, January 14, 2019.

Abb. 35: https://www.facebook.com/rfj.stmk/photos/a.217993564882394/ 1703188516362884/?type=3\&theater, March 6, 2019.

Abb. 36: https://www.facebook.com/fpoe/photos/a.170548806419730/ 1115711805236754/?type=3\&theater, March 10, 2019.

Abb. 37: https://www.facebook.com/fpoe/photos/a.170548806419730/ 866628066811797/?type=3\&theater, March 6, 2019.

Abb. 38: https://www.facebook.com/rfj.stmk/photos/a.217993564882394/ 1788649917816743/?type=3\&theater, March 6, 2019.

Abb. 39: https://www.facebook.com/FJOesterreich/photos/a.307509049850/ $10155306988969851 /$ ?type=3\&theater, March 6, 2019.

Abb. 40: https://www.facebook.com/rfj.stmk/photos/a.217993564882394/ 2324636127551450/?type=3\&theater, March 6, 2019.

Abb. 41: https://www.facebook.com/rfj.stmk/photos/a.217993564882394/ $1820181174663617 /$ ?type=3\&theater, March 6, 2019. 
Abb. 42: https://www.facebook.com/rfj.stmk/photos/a.217993564882394/ 1840674899280911/?type=3\&theate, March 6, 2019.

Abb. 43: https://www.facebook.com/fpoe/photos/a.170548806419730/ 819022324905705/?type=3\&theater, March 6, 2019.

Abb. 44: https://www.facebook.com/fpoe/photos/a.170548806419730/ 820001074807830/?type=3\&theater, March 6, 2019.

Abb. 45: https://www.facebook.com/fpoe/photos/a.170548806419730/ 1098285493646052/?type=3\&theater, March 10, 2019.

Abb. 46: https://www.facebook.com/fpoe/photos/a.170548806419730/ 1121843247956943/?type=3\&theater, March 10, 2019.

Abb. 47: https://www.facebook.com/rfj.stmk/photos/a.217993564882394/ 2237126216302442/?type=3\&theate, March 6, 2019.

Abb. 48: https://www.facebook.com/tomio.cz/photos/a.185333081477515/ 922866577724158/?type=3\&theater, February 10, 2020.

Abb. 49: https://www.facebook.com/tomio.cz/photos/a.185333081477515/ $722169044460580 /$ type=3\&theater, February 10, 2020.

Abb. 50: https://www.facebook.com/hnutispd/photos/a.974781025886076/ 1353956611301847/?type=3\&theater, February 10, 2020.

Abb. 51: https://www.facebook.com/rfj.stmk/photos/a.217993564882394/ 1686429141372155/?type=3\&theater, March 6, 2019.

Abb. 52: https://www.facebook.com/FJOesterreich/photos/a.307509049850/ 10154971634399851/?type=3\&theater, March 6, 2019.

Abb. 53: https://www.facebook.com/tomio.cz/photos/a.185333081477515/ 884506191560197/?type=3\&theater, February 29, 2020.

Abb. 54: https://www.facebook.com/hnutispd/photos/a.974781025886076/ 1629631693734336/?type=3\&theater, February 10, 2020.

Abb. 55: https://www.facebook.com/tomio.cz/photos/a.185333081477515/ 1607636429247166/?type=3\&theater, February 10, 2020.

\title{
Effekte der Affekte. Das politische Spiel mit dem Gemüt des Rezipienten
}

\begin{abstract}
Die immer lauter werdende Stimme des rechtspopulistischen Diskurses verdient ohne Zweifel unsere Aufmerksamkeit. In diesem Zusammenhang drängen sich die Fragen auf: Was verhilft den unterschiedlichen rechts orientierten populistischen Subjekten zu ihrem Erfolg? Womit und wie sprechen sie ihre Wähler an, um deren Stimmen zu gewinnen? Der vorliegende Beitrag setzt sich mit den Techniken und Strategien der politischen Manipulation, insbesondere mit der Technik der Emotionalisierung, auseinander. Im Fokus stehen dabei zwei entgegengesetzte Pole auf der emotionalen bzw. affektiven Skala, die sich mit Trauer und Weinen oder Lachen und Lust assoziieren lassen. Die Untersuchung befasst sich mit dem visuellen Material (Plakate, Onlineauftritte usw.), die Parteien im Rahmen ihrer Öffentlichkeitsarbeit verwenden. Dieses hat das größte und schnellste Potenzial, das Interesse der Rezipienten zu wecken. Analysiert wird, welche Effekte sich aus der Darstellung und Instrumentalisierung der Affekte und Emotionen ergeben und wie sie den Rezipienten beeinflussen können. Schlüsselwörter: Rechtspopulismus, politisches Plakat, Mitleid, Trauer, Weinen, Lachen, politischer Witz, Karikatur, politische Manipulation.
\end{abstract}




\title{
Efekty afektów. Polityczna gra na emocjach odbiorcy
}

\begin{abstract}
Abstrakt: Głos prawicowego dyskursu populistycznego, który staje się coraz głośniejszy, niewątpliwie zasługuje na naszą uwagę. W tym kontekście nasuwają się następujące pytania: co pomaga różnym prawicowym populistom osiągnąć sukces? Z czym i w jaki sposób zwracają się do swojego wyborcy, aby zdobyć jego głos? W artykule omówiono techniki i strategie manipulacji politycznej, w szczególności technikę emocjonalizacji. Skupiono się na dwóch przeciwstawnych biegunach na skali emocjonalnej/afektywnej związane z żalem i płaczem lub śmiechem i pożądaniem. Badanie dotyczy materiałów wizualnych (plakatów, występów online itp.) używanych w public relations i posiadających największy potencjał, by najszybciej dotrzeć i wzbudzić zainteresowanie odbiorcy. Analizie poddano efekty wynikające z reprezentacji i instrumentalizacji afektów i emocji mających wpływać na odbiorcę.
\end{abstract}

Słowa kluczowe: prawicowy populizm, plakat polityczny, współczucie, żal, płacz, śmiech, dowcip polityczny, karykatura, manipulacja polityczna. 